\title{
Practical challenges in patients with stage III NSCLC receiving checkpoint inhibitors after chemoradiation
}

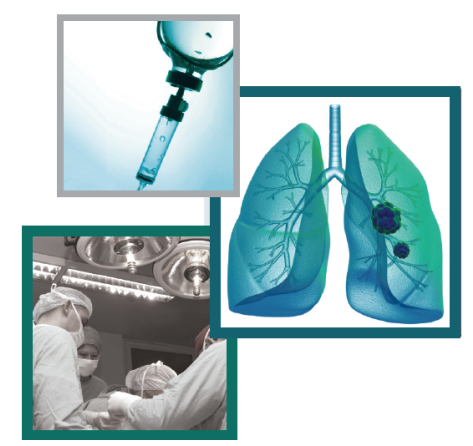

\author{
Nikhil A Shukla*,1 \& Nasser H Hanna ${ }^{1}$ \\ ${ }^{1}$ Division of Hematology/Oncology, Department of Medicine, Indiana University Melvin \& Bren Simon Cancer Center, Indianapolis, \\ IN 46202, USA \\ *Author for correspondence: nashukla@iu.edu
}

\section{"The widespread use of CPI after CRT will result in more patients presenting new and difficult challenges for treating physicians."}

First draft submitted: 17 January 2020; Accepted for publication: 27 January 2020; Published online: 6 February 2020

Keywords: checkpoint inhibitors • chemoradiation • lung cancer

NSCLC accounts for $80 \%$ of all lung cancer diagnoses, and approximately a third of patients present with locally advanced (stage III) disease [1]. The optimal therapy for fit patients with unresectable and/or inoperable stage III disease has evolved over the last three decades from radiation alone to sequential chemoradiation (CRT) to concurrent CRT followed by checkpoint inhibitor therapy (CPI). The recently reported PACIFIC trial generated a paradigm shift in the treatment of such patients, with a 3-year overall survival (OS) of $57 \%$ for patients receiving consolidation durvalumab compared with $43.5 \%$ for those receiving placebo [2-4]. Similar outcomes were reported with consolidation pembrolizumab in a Hoosier Cancer Research Network (HCRN) Phase II Trial [5]. In this article, we explore key clinical challenges that arise when treating patients with consolidation CPI after CRT for patients with stage III NSCLC; namely, the management of CPI-related pneumonitis, timing of consolidation CPI and their role in patients with PD-L1 TPS $<1 \%$ and distinguishing local progression versus pseudoprogression.

\section{Radiation versus checkpoint inhibitor-induced pneumonitis: risk factors \& management}

Prior to the reporting of the PACIFIC trial and the HCRN LUN 14-179 trial, the safety of administering consolidation CPI after CRT was unknown. A primary safety concern with this strategy included the risk of developing life-threatening pneumonitis. CRT alone for the treatment of stage III NSCLC results in a 5-15\% rate of symptomatic pneumonitis. Furthermore, CPI causes symptomatic pneumonitis in approximately $5 \%$ of patients treated for stage IV NSCLC [6,7]. The risk factors for radiation and CPI-induced pneumonitis include the addition of chemotherapy (concurrently with radiation and as consolidation to CRT), higher volume of lung receiving radiation and underlying interstitial lung disease $[8,9]$. Distinguishing CPI-induced pneumonitis from radiationinduced pneumonitis can be challenging. Both treatment modalities cause pneumonitis early (typically within 12 weeks of treatment exposure) and result in similar symptoms of cough, wheezing, dyspnea and fever $[9,10]$. Computed tomography findings may differentiate the likely etiology. Key radiographic features for radiationinduced pneumonitis include localized consolidative ground-glass opacities confined to the radiation treatment fields, whereas CPI-induced pneumonitis may be more diffuse and tend to involve the lower lung fields [11].

Although the initial management of radiation and CPI-induced pneumonitis involves the use of corticosteroids, the dose and duration can differ. Grade 1 pneumonitis constitutes asymptomatic radiographic findings and requires no treatment. Grade 2 CPI-induced pneumonitis is symptomatic but does not require supplemental oxygen support. Patients should be treated with $0.5-1.0 \mathrm{mg} / \mathrm{kg}$ of prednisone until complete resolution of symptoms to baseline. Following symptom resolution, prednisone should be tapered over a minimum of 2 weeks to prevent a rebound of pneumonitis symptoms. In the event of grade 3 or 4 CPI-induced pneumonitis, patients should be treated with $1-2 \mathrm{mg} / \mathrm{kg}$ of prednisone, depending on the severity of the clinical scenario. Such patients should usually be hospitalized, and pulmonary and infectious disease evaluations are warranted as atypical viral and 
fungal infections as well as pre-existing pulmonary diseases such as bronchiolitis obliterans organizing pneumonia, cryptogenic organizing pneumonia and sarcoidosis can have similar clinical presentations. Assuming there is clinical improvement; the dose of prednisone should be maintained until resolution of symptoms and followed by a slow taper over 4 weeks or more. If clinical improvement is not apparent after a few days to a week, infliximab may be administered and repeated in 1-2 weeks [12]. While patients are receiving $>20 \mathrm{mg} /$ day of prednisone, pneumocystis jirovecii prophylaxis should be administered. Once pneumonitis has resolved, patients who experienced grade 1-2 pneumonitis can be re-challenged with CPI. However, we recommend that patients who experience grade $\geq 3$ pneumonitis should not be re-challenged with CPI.

\section{Optimal time to initiate checkpoint inhibitor consolidation}

The optimal time to initiate CPI following CRT in patients with stage III NSCLC is not clearly defined. Patients with stage III NSCLC enrolled in the PACIFIC trial were randomized to durvalumab or placebo within 1-42 days after completing CRT. In an unplanned, retrospective, subset analysis, those patients who received durvalumab $\leq 2$ weeks from completing CRT had improved progression-free survival (PFS; hazard ratio, 0.39; 95\% CI: 0.26-0.58) compared with those who received durvalumab $>2$ weeks after completing CRT (hazard ratio, $0.63,95 \%$ CI: $0.49-0.80)$ [3]. In contrast, the HCRN trial enrolled patients $28-56$ days after CRT to receive consolidation pembrolizumab. An unplanned, retrospective, subset analysis from this trial suggested no difference in any efficacy end point (time to metastatic disease or death, PFS or OS) for those receiving pembrolizumab 4-6 weeks after CRT compared with those receiving it 6-8 weeks after CRT [5]. Therefore, no conclusive statements can be made regarding the optimal timing of CPI after CRT. Those patients who received durvalumab soon after CRT may have been a more fit patient population with fewer toxicities from CRT compared with those patients requiring a longer period of recovery. The HCRN trial results suggest no detriment in delaying CPI up to 8 weeks to recover from toxicities of CRT. The ongoing PACIFIC-2 trial, which randomizes patients with stage III NSCLC to receive concurrent durvalumab plus CRT followed by durvalumab versus CRT followed by durvalumab, will help determine the optimal timing of CPI following CRT [13]. Until such results are known, it is our practice to allow up to 8 weeks for recovery prior to initiating CPI for patients who have experienced significant toxicity to CRT. Those who are more fit and have few toxicities from CRT can safely initiate CPI sooner, including within 2 weeks of completing CRT.

\section{The role of consolidation checkpoint inhibitor therapy in patients with PD-L1 $\leq 1 \%$}

In the PACIFIC trial, approximately $30 \%$ of patients had a PD-L1 TPS of $<1 \%$ [2]. Although there appears to be an increased efficacy for CPI in patients with PD-L1 positive tumors who have stage IV NSCLC, the impact of PD-L1 score on the outcomes of patients with stage III NSCLC receiving CRT followed by CPI is less well defined. The PACIFIC trial demonstrated comparable improvement in PFS and OS in patients with PD-L1 $<25 \%$ versus $\geq 25 \%$. However, in an unplanned subset analysis of patients with PD-L1 $<1 \%$, PFS was superior for those who received consolidation durvalumab $(\mathrm{p}=$ not significant), while OS favored those receiving placebo ( $\mathrm{p}=$ not significant) [3]. Based on this finding, the European Medical Agency (Amsterdam, Netherlands) has approved consolidation durvalumab only for patients with tumors expressing PD-L1 $\geq 1 \%$. The US FDA has no such restriction. In contrast to durvalumab on the PACIFIC trial, the efficacy of consolidation pembrolizumab in the HCRN trial was similar for patients regardless of PD-L1 score [5]. Our practice is to treat patients with consolidation CPI after CRT, regardless of PD-L1 status on the initial tumor biopsy. We believe that baseline tumor PD-L1 score may not be an accurate indicator of consolidation CPI efficacy. CRT increases the activity of cytotoxic $\mathrm{T}$ cells and upregulates expression of PD-L1 on tumor cells (including on cells that are PD-L1 negative), thereby sensitizing tumors to CPI [14]. Furthermore, discordant PD-L1 scores have been reported between primary tumor and metastatic sites or regional lymph nodes [15]. Finally, there is an increasing evidence that other biomarkers may also be predictive of response to CPI, including tumor mutational burden, CD4/8 ratios and even individual gene mutations such as STK11 [16].

\section{Distinguishing true progression from pseudoprogression}

As the use of consolidation CPI after CRT becomes routine, physicians will be challenged in interpreting radiographic changes that evolve over time. Pseudoprogression is an established phenomenon that occurs in approximately $5 \%$ of patients receiving CPI [17]. Distinguishing pseudoprogression from evolving radiation fibrosis or localized true progression is difficult. Pseudoprogression is usually transient and not associated with clinical deterioration. 
Therefore, caution should be heeded in declaring patients to have progressive disease and prematurely initiating salvage interventions. It is our practice to document pathological recurrence or clear and convincing clinical progression before initiating salvage therapy. Utilizing the iRECIST criteria, rather than RECIST is essential when considering whether a patient has experienced treatment failure. The iRECIST criteria require confirmation of progressive disease 4 weeks after the initial signs of progression or new disease [18].

Consolidation CPI following CRT has resulted in unprecedented increases in survival outcomes for patients with stage III NSCLC. The widespread use of CPI after CRT will result in more patients presenting new and difficult challenges for treating physicians. Key challenges are outlined in this article. In summary, we recommend vigilance in recognizing CPI-related pneumonitis and early consultation with pulmonologists and infectious disease physicians as other pulmonary diseases and infections can masquerade as pneumonitis. Patients with grade 2 or higher pneumonitis should not have steroids tapered until clinical symptoms have resolved or return to baseline. Second, there is no conclusive evidence that delay of initiating CPI for up to 8 weeks to allow recovery from CRT toxicities is detrimental. Third, we believe patients with tumors that are PD-L1 $<1 \%$ should not be excluded from receiving consolidation CPI after CRT. At last, caution should be heeded when declaring a patient to have locally progressive disease after CRT and CPI as radiographic findings may be misleading.

Financial \& competing interests disclosure

Nasser Hanna has received grant money for investigator-initiated clinical trials from Merck, AstraZeneca, Bristol Myers Squibb, and Genentech. He has received renumeration from "Up to Date" for serving as the editor for the section on adjuvant therapy in lung cancer and has also served as a member of a Data Safety Monitoring Board for Beyond Spring. The authors have no other relevant affiliations or financialinvolvement with any organization or entity with a financial interest in or financial conflict with the subject matter or materials discussed in the manuscript apart from those disclosed.

No writing assistance was utilized in the production of this manuscript.

\section{Open access}

This work is licensed under the Attribution-NonCommercial-NoDerivatives 4.0 Unported License. To view a copy of this license, visit http://creativecommons.org/licenses/by-nc-nd/4.0/

\section{References}

1. Ettinger DS, Akerley W, Borghaei H et al. Non-small cell lung cancer. J. Natl Compr. Cancer Netw. 10(10), 1236-1271 (2012).

2. Antonia SJ, Villegas A, Daniel D et al. Durvalumab after chemoradiotherapy in stage III non-small-cell lung cancer. N. Eng. J. Med. 377(20), 1919-1929 (2017).

3. Antonia SJ, Villegas A, Daniel D et al. Overall survival with durvalumab after chemoradiotherapy in stage III NSCLC. N. Eng. J. Med. 379(24), 2342-2350 (2018).

4. Gray JE, Villegas A, Daniel D et al. Three-year overall survival update from the PACIFIC trial. J. Clin. Oncol. 37 (Suppl. 15), 8526 (2019).

5. Durm GA, Althouse SK, Sadiq AA et al. Phase II trial of concurrent chemoradiation with consolidation pembrolizumab in patients with unresectable stage III non-small cell lung cancer: Hoosier Cancer Research Network LUN 14-179. J. Clin. Oncol. 36(Suppl. 15), 8500 (2018).

6. Nishino M, Giobbie-Hurder A, Hatabu H, Ramaiya NH, Hodi FS. Incidence of programmed cell death 1 inhibitor-related pneumonitis in patients with advanced cancer: a systematic review and meta-analysis. JAMA Oncol. 2(12), 1607-1616 (2016).

7. Jain V, Berman AT. Radiation pneumonitis: old problem, new tricks. Cancers (Basel) 10(7), E222 (2018).

8. Kong FM, Wang S. Non-dosimetric risk factors for radiation-induced lung toxicity. Semin. Radiat. Oncol. 25(2), 100-109 (2015).

9. Naidoo J, Wang X, Woo KM et al. Pneumonitis in patients treated with anti-programmed death-1/programmed death ligand 1 therapy. J. Clin. Oncol. 35(7), 709-717 (2017).

10. Choi Y, Munden RF, Erasmus JJ et al. Effects of radiation therapy on the lung: radiologic appearances and differential diagnosis. Radiographics 24(4), 985-997 (2004).

11. Schoenfeld JD, Nishino M, Sevegnini M, Mak RH, Hodi FS. Pneumonitis resulting from radiation and immune checkpoint blockade illustrates characteristic clinical, radiologic and circulating biomarker features. J. Immunother. Cancer 7(1), 112 (2019).

12. Chuzi S, Tavora F, Cruz M et al. Clinical features, diagnostic challenges, and management strategies in checkpoint inhibitor-related pneumonitis. Cancer Manag. Res. 9, 207-213 (2017). 
13. Bradley JD, Nishio M, Okamoto I et al. PACIFIC-2: Phase III study of concurrent durvalumab and platinum-based chemoradiotherapy in patients with unresectable, stage III NSCLC. J. Clin. Oncol. 37(Suppl. 15), TPS8573 (2019).

14. Deng L, Liang H, Burnette B et al. Irradiation and anti-PD-L1 treatment synergistically promote antitumor immunity in mice. J. Clin. Invest. 124(2), 687-695 (2014).

15. Munari E, Zamboni G, Marconi M et al. PD-L1 expression heterogeneity in non-small cell lung cancer: evaluation of small biopsies reliability. Oncotarget 8(52), 90123-90131 (2017).

16. Havel JJ, Chowell D, Chan TA. The evolving landscape of biomarkers for checkpoint inhibitor immunotherapy. Nat. Rev. Cancer 19(3), 133-150 (2019).

17. Katz SI, Hammer M, Bagley SJ et al. Radiologic pseudoprogression during anti-PD-1 therapy for advanced non-small-cell lung cancer. J. Thorac. Oncol. 13(7), 978-986 (2018).

18. Vrankar M, Unk M. Immune RECIST criteria and symptomatic pseudoprogression in non-small-cell lung cancer patients treated with immunotherapy. Radiol. Oncol. 52(4), 365-369 (2018). 